\title{
A case report of gastric cancer with brain metastasis: Rare peripheral nervous system symptoms
}

\author{
GE-LIANG YANG ${ }^{1}$, TIAN-HANG LUO ${ }^{2}$, HUI-QING ZHANG ${ }^{1}$, CHANG-QUAN LING $^{1}$ and BAI LI ${ }^{1}$ \\ Departments of ${ }^{1}$ Integrative Oncology and ${ }^{2}$ General Surgery, Changhai Hospital, \\ Second Military Medical University, Shanghai 200433, P.R. China
}

Received December 24, 2014; Accepted January 26, 2016

DOI: $10.3892 / \mathrm{ol} .2016 .4288$

\begin{abstract}
Gastric cancer with brain metastasis is rare. The present study reports a case of gastric cancer with isolated brain metastasis 1 year after gastrectomy. To the best of our knowledge, there have been no prior reports of solitary brain metastasis from gastric cancer with peripheral nervous system symptoms. A distal gastrectomy was performed on a 60-year-old male patient with gastric cancer in November 2012. Postoperative pathological analysis revealed a moderately differentiated adenocarcinoma with tumor invasion into the serosa and metastasis to one dissected lymph node. No abnormalities were found at follow-up examination. However, a tumor representing metastasis to the brain was recognized by a cranial enhanced magnetic resonance imaging examination 1 year after gastrectomy, which was performed when the patient exhibited numbness and thigmesthesia. The patient was administered $30 \mathrm{~Gy}$ of stereotactic radiotherapy, delivered in 5 fractions. The patient succumbed to disease 10 months subsequent to undergoing radiotherapy. This case report suggests that gastric cancer may re-present as brain metastasis with peripheral nervous system symptoms.
\end{abstract}

\section{Introduction}

Gastric cancer is one of the most widespread malignant tumors in the world. According to the latest world cancer statistics, 700,000 patients succumbed due to gastric cancer in 2012, making it the third most common cause of cancer mortality (1). In 2005, 300,000 mortalities and 400,000 new cases of gastric cancer were reported in China (2). Metastasis from gastric cancer typically occurs in the abdominal cavity, peritoneum, lymph nodes and liver (3). Metastasis limited to

Correspondence to: Professor Bai Li or ProfessorChang-QuanLing, Department of Integrative Oncology, Changhai Hospital, Second Military Medical University, 168 Changhai Road, Shanghai 200433, P.R. China

E-mail: libai9@126.com

E-mail: lingchangquan@gmail.com

Key words: gastric cancer, brain metastasis, peripheral nervous system symptoms the brain is rare and prognosis in such cases is poor $(4,5)$. It has been reported in $<1 \%$ of clinical cases, the majority of which are included in 2 large, single-institution retrospective studies. Between 1957 and 1997, a total of 3320 patients were diagnosed with gastric cancer at M.D. Anderson Cancer Center (Houston, TX, USA), and only 24 of these patients $(0.7 \%)$ were observed to have brain metastases following imaging studies or autopsy. The median survival time was $\sim 9$ weeks, and in certain patients surgical resection followed by whole-brain radiation therapy was associated with relatively long survival times of 54 weeks (5). Another study identified brain metastasis in only 11 out of 2322 patients $(0.47 \%)$ treated between 1980 and 1998 (6). York et al (5) noted neurological improvement in only 4 out of 24 patients $(16.7 \%)$ treated with either surgical resection with whole brain radiation therapy (WBRT) $(n=3)$ or WBRT alone $(n=1)$. The median survival time among the WBRT group did not differ from patients that had received steroid monotherapy. Kaskura et al (4) reported a median survival time of 24.0 weeks in patients that had undergone surgery or surgery with WBRT compared with 10.8 weeks in patients that received WBRT alone. To the best of our knowledge, there have been no prior reports of solitary brain metastasis from gastric cancer with peripheral nervous system symptoms (7-10). The current study presents a rare case of gastric cancer with brain metastasis and nervous system symptoms treated with stereotactic radiotherapy.

\section{Case report}

A 60-year old male patient presenting with nausea, vomiting and epigastric pain for 10 days and was admitted to Shanghai Changhai Hospital affiliated to the Second Military Medical University (Shanghai, China) on November 8, 2012. The patient ate a balanced diet without previous history of peptic ulcer or other gastrointestinal disorders and no family medical history of gastric cancer. Physical examination revealed no abnormalities. Laboratory examinations identified positive carcinoembryonic antigen (CEA) at $310.23 \mathrm{ng} / \mathrm{ml}$ (normal range, $<0.5 \mathrm{ng} / \mathrm{ml}$ ); all other laboratory examinations were negative. Gastric endoscopy revealed a protruding tumor measuring $3.0 \times 2.5 \mathrm{~cm}$ on the anterior wall of the fundus of the stomach. An enhanced computed tomography (CT; Brilliance 16; Philips Medical Systems, Inc., Bothell, WA, USA) scan confirmed a gastric antrum tumor. A distal 


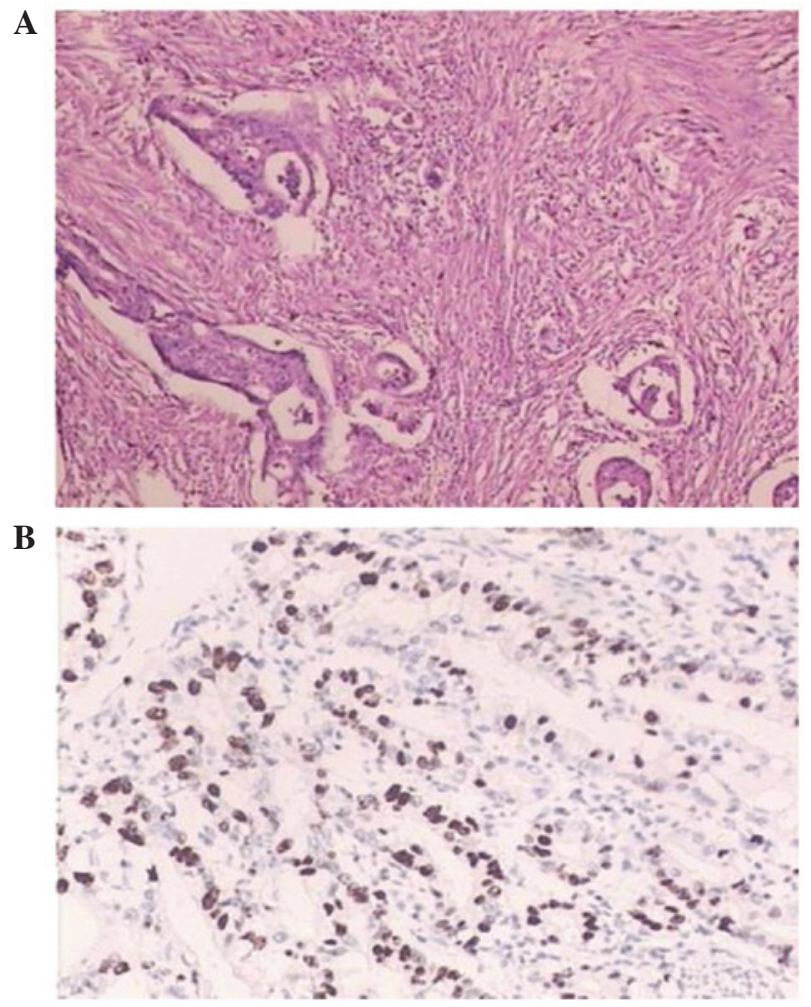

Figure 1. Histological pathology and immunohistochemistry of the stomach in November 2012. (A) Histological pathology of the primary lesion of stomach: revealed moderately differentiated tubular adenocarcinoma (hematoxylin and eosin staining; magnification, x100) and (B) Immunohistochemistry revealed c-erbB-2 (+), p53 (-), epidermal growth factor receptor (-), p16 (-), type II topoisomerase (++) and Ki-67 (60\%).

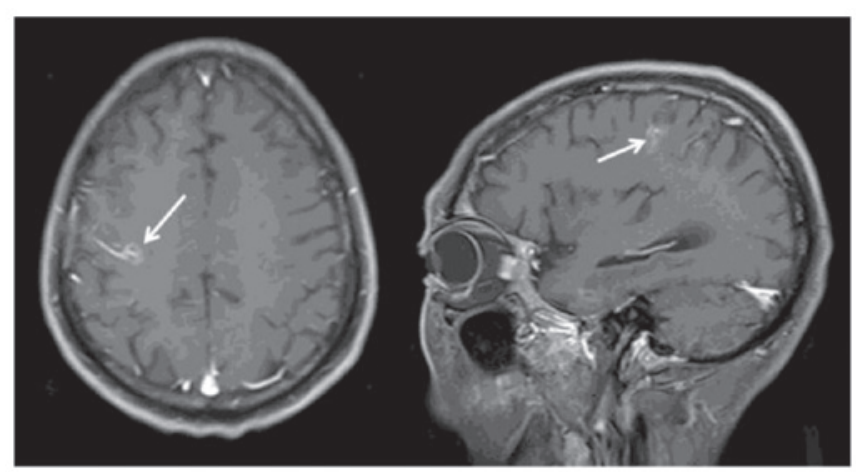

Figure 2. Axial (left) and sagittal (right) enhanced T1-weighted magnetic resonance imaging, revealing an enhanced tumor in the right parietal lobe (white arrow) in November 2013.

gastrectomy was performed on the patient on November 13 , 2012. Postoperative pathological analysis indicated moderately differentiated adenocarcinoma of the stomach antrum, tumor invasion into the serosa and evidence of metastasis into only one of the dissected lymph nodes at the greater curvature. Pathological and immunohistochemical analyses were conducted using the hematoxylin-eosin staining (HE Stain kit; Beyotime Institute of Biotechnology, Haimen, China) and avidin-biotin-peroxidase (Vectastain ABC IHC kit; Vector Laboratories, Inc., Burlingame, CA, USA) methods, respectively. All paraffin sections were $4 \mu \mathrm{m}$ in thickness
(RM2125 RTS The Essential Microtome; Leica Microsystems, Inc., Buffalo Grove, IL, USA). The antibodies used were as follows: Rabbit polyclonal anti-human c-ErbB-2 (dilution, 1:200; catalog no., ab2428); Abcam, Cambridge, MA, USA), mouse monoclonal anti-human p53 (dilution, 1:1,000; catalog no., ab26; Abcam), rabbit polyclonal anti-human epidermal growth factor receptor (EGFR; dilution, 1:2,500; catalog no., ab2430; Abcam), rabbit monoclonal anti-human p16 (dilution, 1:2,000; catalog no., ab51243; Abcam), rabbit polyclonal anti-human type II $\alpha$ topoisomerase (dilution, 1:1,000; catalog no., \#4733; Cell Signaling Technology, Inc., Danvers, MA, USA) and rabbit polyclonal anti-human Ki-67 (dilution, 1:1,000; catalog no., ab15580; Abcam). Immunohistochemical results were as follows: c-erbB-2 (+), p53 (-), EGFR (-), p16 (-), type II topoisomerase (++), Ki-67 (60\%) (Fig. 1).

The postoperative course was uneventful. The patient was discharged in good general condition 1 week after the operation. Between 3 weeks and 5 months after the surgery, the patient received 6 courses of adjuvant chemotherapy according to the SOX scheme $(60 \mathrm{mg}$ oral S-1 twice a day on days 1-14 and $200 \mathrm{mg}$ intravenous infusion oxaliplatin on day 1 every 3 weeks). The patient's CEA levels gradually declined to within normal limits 7 months after the surgery. The patient was systemically followed once every 3 months following the completion of chemotherapy and no abnormalities were noted.

However, 8 months after the surgery, the patient suddenly developed paroxysmal finger numbness of the left hand with hypopselaphesia, and these symptoms did not improve even following nerve nutrition therapy $(0.5 \mathrm{mg}$ oral mecobalamin 3 times a day, for 2 months). Symptoms of numbness were still present 1 year after surgery, with thigmesthesia and loss of precision grip function, accompanied by paroxysmal tinnitus of the left ear. Physical examination revealed attenuated proximal muscle strength (grade IV) (11), decreased deep sensation of the left upper limb and positive Hoffmann sign of the left hand. Cranial enhanced magnetic resonance imaging (MRI; MAGNETOM Avanto 1.5T; Siemens Heathcare, Erlangen, Germany) identified a tumor $(22 \times 15 \mathrm{~mm})$ in the junctional zone of the right frontal lobe and the parietal lobe, accompanied by mild hemorrhage and peritumoral edema (Fig. 2). A lumbar puncture biopsy was performed, however, no malignant cells were found in the cerebrospinal fluid. An enhanced CT scan of the chest and an enhanced MRI scan of the liver demonstrated no abnormalities. Laboratory data were within normal limits, with the exception of signs of anemia, with a red blood cell count of $3.37 \times 10^{12} / 1$ (normal range, $4.0-5.5 \times 10^{12} / 1$ ) and a hemoglobin level of $6.7 \mathrm{~g} / \mathrm{dl}$ (normal range, 12-16 g/dl). The patient was followed up monthly for anemia; however, for $\sim 3$ months there were no changes from the original values.

The patient declined brain metastasectomy or palliative chemotherapy, but received $30 \mathrm{~Gy}$ of stereotactic radiotherapy delivered in 5 fractions over 1 week. A follow-up brain CT performed 2 months after radiotherapy revealed a reduced tumor in the brain $(16 \times 12 \mathrm{~mm})$. The patient was alive with good health condition 8 months after radiotherapy. At this point, the patient's nervous system symptoms were not markedly improved and the patient continued to exhibit anemia (hemoglobin, 6-7 g/dl), which was treated with Yang Xue Yin (10 $\mathrm{ml}$ orally administered 3 times/day). However, the patient 
refused to undergo any further imaging examinations due to the high cost.

\section{Discussion}

The incidence of brain metastasis with gastric cancer is rare and has been reported to be $<1 \%$ of gastric cancer cases $(4,5)$. Furthermore, the majority of the reported cases of brain metastasis were accompanied by lymph node, liver, lung or bone metastasis $(4,5,12)$; there have been extremely few reports of brain metastasis as a solitary lesion from gastric cancer. An early follow-up study reported that the most frequent initial symptoms of brain metastasis are headache, mental status changes, migraine, vision disorder and Jacksonian epilepsy (13). In the present study, these peripheral nervous system symptoms were initially considered to be caused by chemotherapy with oxaliplatin. After obtaining a medical history, and performing a comprehensive physical examination and cranial enhanced MRI, the patient was clinically diagnosed with brain metastasis of gastric cancer. However, malignant tumor cells were not recovered by lumbar puncture to confirm a pathological diagnosis. CEA in cerebrospinal fluid was positive.

A follow-up brain CT performed 2 months after radiotherapy revealed a reduced tumor in the brain. The patient was alive with good health condition 8 months after radiotherapy. The median survival of patients with metastatic gastric cancer is no more than 1 year (13). In particular, the overall prognosis of patients with brain metastases from gastric cancer is extremely poor, with a median survival time of 2-4 months $(4-6,14)$. In the absence of clear guidelines on therapy, each case requires individual analysis and a multidisciplinary approach. Patients with a solitary metastasis are predominantly treated by resection, radiotherapy and chemotherapy, whereas palliative care may be a more appropriate option for the treatment of multiple metastases $(4,5,15,16)$. In the present case, the patient declined brain metastasectomy or palliative chemotherapy and received 30 Gy of stereotactic radiotherapy delivered in 5 fractions.

The present study reports a rare case of gastric cancer with brain metastasis. The present case report highlights several key learning points. Gastric cancer with metastasis may appear as a single lesion in brain and without typical central nervous system symptoms. Peripheral nervous system symptoms should be highlighted for brain metastasis. Thus, the current case raises awareness that gastric cancer may present as brain metastasis, particularly in patients with peripheral nervous system symptoms.
In conclusion, brain metastasis of gastric cancer is difficult to treat. In the current case, the patient rejected brain metastasectomy and chemotherapy. In such cases, stereotactic radiotherapy may be proposed with curative intent when appropriate. The current patient is an outstanding example of the beneficial use of stereotactic radiotherapy for longer survival time.

\section{References}

1. World Health Organization: Latest World Cancer Statistics. Global cancer burden rises to 14.1 million new cases in 2012: Marked increase in breast cancers must be addressed. Press release no. 223. IARC, Lyon/Geneva: Dec 12, 2013.

2. Yang L: Incidence and mortality of gastric cancer in China. World J Gastroenterol 12: 17-20, 2006.

3. Deng J, Liang H, Wang D, Sun D, Pan Y and Liu Y: Investigation of the recurrence patterns of gastric cancer following a curative resection. Surg Today 41: 210-215, 2011.

4. Kasakura Y, Fujii M, Mochizuki F, Suzuki T and Takahashi T: Clinicopathological study of brain metastasis in gastric cancer patients. Surg Today 30: 485-490, 2000.

5. York JE, Stringer J, Ajani JA, Wildrick DM and Gokaslan ZL: Gastric cancer and metastasis to the brain. Ann Surg Oncol 6: 771-776, 1999.

6. Go PH, Klaassen Z, Meadows MC and Chamberlain RS: Gastrointestinal cancer and brain metastasis: A rare and ominous sign. Cancer 117: 3630-3640, 2011.

7. Kitayama Y, Yoden Y and Okamoto N: A case of effective paclitaxel therapy for gastric cancer with brain metastasis. Gan To Kagaku Ryoho 33: 981-984, 2006 (In Japanese).

8. Kojima Y, Watanabe T, Sanada T, Hiramatsu Y, Nakane Y, Yamamura M, Hioki K and Yamamoto M: A case report of brain metastasis due to an advanced gastric cancer. Gan No Rinsho 34: 1731-1734, 1988 (In Japanese).

9. Mizumatsu S, Nishimura T, Sakai K, Goto M, Sugatani H and Higashi T: A case of brain metastasis from gastric cancer involving bilateral middle cerebellar peduncles. No Shinkei Geka 34: 955-960, 2006 (In Japanese).

10. Perri F, Bisceglia M, Giannatempo GM and Andriulli A: Cerebellar metastasis as a unique presenting feature of gastric cancer. J Clin Gastroenterol 33: 80-81, 2001.

11. James MA: Use of the Medical Research Council muscle strength grading system in the upper extremity. J Hand Surg Am 32: 154-156, 2007.

12. Chang YR, Han DS, Kong SH, Lee HJ, Kim SH, Kim WH and Yang HK: The value of palliative gastrectomy in gastric cancer with distant metastasis. Ann Surg Oncol 19: 1231-1239, 2012.

13. Stortebecker TP: Metastatic tumors of the brain from a neurosurgical point of view; a follow-up study of 158 cases. J Neurosurg 11: 84-111, 1954

14. Nomura T, Yoshikawa T, Kato H, Nikkuni K, Sasaki K, Shirai Y and Hatakeyama K: Early gastric cancer manifested as brain metastasis: Report of a case. Surg Today 27: 334-336, 1997.

15. Murawa D, Nowaczyk P, Szymkowiak M and Karaszewska B: Brain metastasis as the first symptom of gastric cancer-case report and literature review. Pol Przegl Chir 85: 401-406, 2013.

16. Bussing A, Zhai XF, Peng WB and Ling CQ: Psychosocial and spiritual needs of patients with chronic diseases: Validation of the Chinese version of the spiritual needs questionnaire. J Integr Med 11: 106-115, 2013. 